\title{
Intelligent Farmland Management System with Low-power Consumption Based on the Internet of Things
}

\author{
Zhigang Wang ${ }^{1}$ \\ ${ }^{1}$ Huanghe Science \& Technology College, Zhengzhou, Henan province, China
}

Keywords: Internet of things, Intelligent agriculture, Low-power consumption, Information acquisition .

Abstract. The global demand for green technology and energy efficiency is driving the development of a new intelligent agriculture with low-power consumption based on the Internet of things. This kind of Internet is constantly being used in intelligent agriculture. The new wireless network can work for a longer time with battery. In the life cycle of the application, it requires little or no maintenance. Therefore, reducing node power consumption becomes the difficulty and key point of the Internet of things. Because this system is a scientific research, which needs constant practice. We need to do experiments seriously and solve the problems timely. In this way, we can make an economical and practical intelligent agricultural monitoring system with low-power consumption based on the Internet of things to serve the agricultural industry.

\section{Introduction}

The global demand for green technology and energy efficiency is driving the development of a new intelligent agriculture with low-power consumption based on the Internet of things. This kind of Internet is constantly being used in intelligent agriculture. The new wireless network can work for a longer time with battery. In the life cycle of the application, it requires little or no maintenance. Therefore, reducing node power consumption becomes the difficulty and key point of the Internet of things. Low- power consumption is mainly composed of wireless network, low-power node query system and low-power node processor. The low-power consumption of nodes is divided into hardware low-power consumption and software low-power consumption. There are four major wireless communication modes: send mode, receive mode, idle mode and sleep mode. The power consumption mainly focus on the first three. The start-up power consumption is also very high.

In recent years, with the rapid development of computer technology and wireless sensor technology, the intelligent agriculture based on the Internet of things has attracted more and more attention from experts and scholars. The Internet of things has been rapidly promoted and applied in the intelligent agriculture, especially for the greenhouse. Environmental factors in the greenhouse such as temperature, humidity, illumination, $\mathrm{CO}_{2}$ concentration have great influence on crop production. Traditional way of manual control is hard to meet the requirements of scientific and rational planting. At present, the system of automatic monitoring of the above environmental factors is not widely available in China. What's more, because of the expensive price, it is not suitable to import the control system of large multi-span greenhouse with multifunction from foreign countries. Here are some advantages of the system mentioned in this paper comparing with the intelligent agricultural monitoring systems at home and abroad.

The application of low-power consumption technology. According to the working state of the node, there are three states: "Operation", "Dormancy" and "Deep dormancy". The "Operation" mode starts when it is collecting information, and the other time is in the modes of "Dormancy" and "Deep dormancy", which effectively reduces the power consumption of the node.

The efficient application of wireless self-organizing mesh network on the intelligent agriculture. The networking of wireless self-organizing mesh network and node control technology are applied to the node communication of the Internet of things, which effectively improved the node safety. Since nodes are net transmission, each node can be transmitted as a relay. While there may be individual node damaged, other nodes can communicate by passing the damaged node. 
The application of Internet of things technology on intelligent agriculture can achieve both the refined management and control of agricultural science and the further improvement of labor efficiency.

Because this system is a scientific research, which needs constant practice. We need to do experiments seriously and solve the problems timely. In this way, we can make an economical and practical intelligent agricultural monitoring system with low-power consumption based on the Internet of things to serve the agricultural industry.

Through the scientific research, a kind of information acquisition system which takes intelligent agriculture monitoring as the core and is easy to operate will be developed to save the monitoring costs for agricultural production. Thus the cost of agricultural products will be reduced.

The Internet of things of intelligent agriculture and the node acquisition system with low-power consumption are mainly connected by the temperature sensor, the soil sensor, the humidity sensor and the temperature controller, the humidity controller, air tester, and the automatic sprinkler irrigation system. Through WIFI device server, the data such as temperature, humidity, soil aridity can be delivered to the remote intelligent system in real time. Then the data will be sent through mobile phones or handheld terminals to agricultural workers and experts, which will provide data basis to their remote guidance and scheme decision. Then the guidance will be sent to the farmers to help them manage the farmland.

The data center analyzes the environmental parameters of the greenhouse, which are collected in real time. Such as temperature, humidity, illumination, soil temperature, soil humidity, $\mathrm{CO}_{2}$ concentration, leaf wetness, dew point temperature. The data center not only makes the corresponding statistical reports, but also makes the trend analysis, and show them to the users by intuitive graphs and curves. The center also provide all kinds of sound and light alarm information according to the requirements of crops. For example, when the temperature and humidity exceed the set value, the system will automatically turn on or off the temperature controller, the humidity controller, or the automatic sprinkler irrigation equipment, etc. Then the monitoring center will send the data through mobile phones or handheld terminals to agricultural workers and experts, which will provide data basis to their remote guidance and scheme decision. Then the guidance will be sent to the farmers to help them manage the farmland.

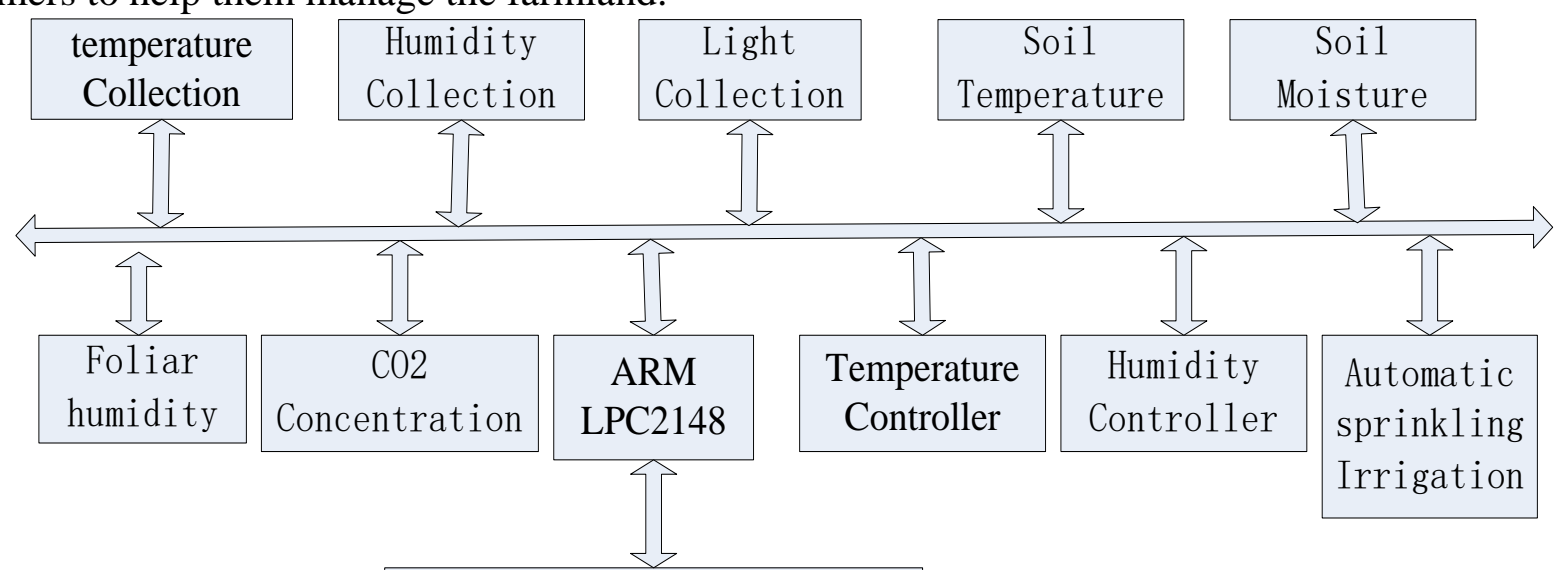

Radio Transmit Receive Node

Fig. 1 The block diagram of Wireless transceiver module hardware

The collection of environmental parameters in the greenhouse is controlled by ARMLPC2148. Such as temperature, humidity, illumination, soil temperature, soil humidity, $\mathrm{CO}_{2}$ concentration, leaf wetness, dew point temperature. It also controls the temperature controller, the humidity controller, and automatic sprinkler irrigation system. The collected data is launched out through the wireless module and received commands from the control center.

To study this problem from the angle of the single-chip microcomputer(SCM), we first need to discuss the various power consumption patterns of the Internet of things. According to the processing demand, the power consumption modes of an SCM should have a distinct set of preset working modes. An embedded SCM can use one of its peripherals to sample the signal of the surrounding 
environment. It may not have other things to do before it collects a certain number of samples. Then the SCM may be in "Dormancy" or in a ultra-low power standby mode between sampling each data. Once the application has read enough data, the SCM can easily switch to "Full-speed operation" mode. It means that the SCM is woken up and running at its maximum speed. An SCM usually receives some types of evocation events so that it exits from various low-power consumption modes. The evocation events can be triggered by external excitation signals, such as an I/O pin level flipping, or internal processor activities, such as an interrupt event triggered by a timer peripheral. The specific power consumption modes supported by SCMs are different, but usually these modes have something in common. The typical power consumption modes are as follows:

The "Always run" mode

The "Dormancy" or "Standby" mode (maintain power to the memory)

The "Deep dormancy" mode (the power of the memory is off to minimize power consumption)

When designing the Internet of things, the low-power consumption mode of the SCM should be especially noticed. The design method with minimum power consumption is the best. While designing, the SCM should run in the mode of "Dormancy" or "Deep dormancy" for most of the time. The power consumption goes to the maximum when the processor works at full speed; The power consumption is relatively low when the processor is in its standby mode. There are two common standby modes: idle mode and shutdown mode. The idle mode can exit through interruptions, which can be supplied by external events. The shutdown mode means that the processor stops and doesn't respond even when there is an interruption. Thus it can only exit from the mode while resetting. In order to reduce the power consumption of the system, once the CPU is in "idling", it can enter idle mode and reduce power consumption. If an external event occurs during the period, an interrupt signal can be generated by the event, allowing the CPU to enter the running state. For the shutdown mode, the CPU can only be waken with a reset signal. Compiler optimization is another effective way to reduce power consumption. The compiler's role is to translate programs written in high-level languages, such as $\mathrm{C} / \mathrm{C}++$, into programs that can be executed on the target machine. In other words, the compiler provides an abstraction layer for high-level language programmers, which allows the programmers to write high level language codes that is close to the actual problems (instead of assembler or machine language) to solve actual problems conveniently. At the same time, the program's readability and maintainability are guaranteed to improve the efficiency of software development. In addition, if the program needs to be put in a new target machine, the program only need to be recompiled with the corresponding compiler instead of being rewritten. But in some cases, this is done at the expense of the execution performance of the program. The effectiveness of the compiler and the code efficiency it generates can be compared to the codes written by the assembler in an expert-level /machine language programmer. So codes with better efficiency can be generated by optimizing the compiler. In a word, optimizing the compiler can effectively reduce the power consumption of embedded devices.

There is a network organizing method of the self-organizing network for the Internet of things monitoring system. For the wireless data access system, which is composed of the information acquisition node, the information sending node and the information acquisition center, the steps of the self-organizing network include:

Information acquisition node is used for information collection. After powered on, the information acquisition node is used to collect strapping table address of environmental parameters like temperature, humidity, illumination, soil temperature, soil humidity, $\mathrm{CO}_{2}$ concentration, leaf wetness, dew point temperature and so on. When reading successfully, the information address of the strapping table will be replaced with physical address. After that, the network preparation of the information acquisition node is ready.

The information sending node is used for sending information. It receives the network beacons issued by the information acquisition center and the field intensity collection instruction and signals; It receives the network beacon instruction to determine whether it is sent by the specified channel or not. If yes, it will record the field intensity and determine if it exceeds the limit value of the forwarding. If the field intensity does not exceed the limit value, then the node will forward the 
network beacon instruction; It receives the field intensity collection instruction and determines whether it is legal or not. If yes, it will determine whether the instruction is the relay. If yes, the field intensity acquisition instruction will be forwarded. Otherwise, the node will have feedback based on the instruction. Then the information acquisition center forms a configuration table for information sending nodes to establish the routing.

After receiving the configuration table of the information acquisition center and the physical address of the information acquisition node confirmed by the information acquisition center, the information sending center will send the feedback to the information acquisition center. The information acquisition center then downloads the ID mapping table of the information acquisition node from the central control platform, and then instructs the information acquisition node to build the network after determining that all the information sending nodes are online.

When the information acquisition node is powered on, it will read the strapping table address. After that, it can replace the strapping table address with physical address. The network preparation of the information acquisition node is ready. Then the main channel group or the designated channel group will be scanned. Then comes to the field intensity collection stage. When the information acquisition node is in the network, it will scan the designated channel group. After receiving the field intensity collection instruction sent by the information acquisition center, it can determine whether the instruction is legal or not. If yes, then it will continue to judge whether it is the relay or not. If yes, it will continue to forward the field intensity collection instruction according to the relay address. If no, it will determine whether the destination address is the information acquisition center. If yes, according to the field intensity collection instruction, it will have a feedback to assist the information acquisition center to collect the field intensity.

The characteristics of the network organizing method of the self-organizing network for the wireless data information acquisition system is that: When the information acquisition node is not connected, the information acquisition node is first scanned by the main channel group. After receiving the networking beacons forwarded by the information acquisition and sending center, it can determine whether they are sent by the specified channel or not. If yes, it will record the corresponding field intensity and determine if it exceeds the limit value of the forwarding. When judging, according to its own field intensity signal, the information acquisition node uses the Internet of things protocol to calculate the timeslot number and the number of forwarding turns. Then, according to the calculation results, if the maximum number of forwarding turns (the limit value) is reached, the network beacon will not be forwarded. Otherwise, the carrier sense protocol can be used for carrier sense, and the network beacon will be forwarded when the channel is idle. If the channel is busy, the beacon can be forwarded until the next turn. The information sending node sends the node query information, and the information receiving node receives the information to set up the main node, build the query list of the main node, and query all nodes in the region one by one to build the query list of the slave nodes. After that, the communication between nodes has been completed.

In the information sending node and information acquisition node, it is set up as the activation method of the internal dormancy. All nodes begin as dormancy mode, and the main node can query all nodes in the region according to the strength of the field intensity signal, and form a node distribution diagram. When the information sending node sends the acquisition information, it changes from dormancy into active state, and sends the node query information. When the information acquisition node receives the query information sent by the information sending node, it changes from dormancy to active state, collects and sends the information. After sending the information, the information acquisition node will change from the active state to dormancy. If the information sending node doesn't receive the information from the information acquisition node in a certain time, it will also change to the dormancy state and record the information acquisition node as "lost".

Through the use of intelligent farmland management system based on the Internet of things, the basic information of farmland can be quickly obtained, which includes the temperature, humidity, irrigation, and the upward trend of agricultural products and so on. It is beneficial to the market sales of agricultural products, which can help farmers to a great extent. Through the research and use of this 
project, it can be found that the Internet of things has a very good application prospect in intelligent agriculture.

About the author: Zhigang Wang, an associate professor and master of Huanghe Science \& Technology College. Research direction: The application of embedded technology in communication.

\section{Acknowledgement}

Fund: The cloud application engineering research center of intelligent agriculture based on the Internet of things in Henan.

\section{References}

[1] Jianmin Zhou, Hongyan Yin, Dongdong Xu. Greenhouse Environment Monitoring System Based on Zigbee Technology [J]. Instrument Technique and Sensor,2011(9):50-52

[2] Qibo Sun, Jie Liu. Internet of Things: A Research Overview of Concepts, Structures and Key Technologies [J]. Journal of Beijing University of Posts and Telecommunications, 2010(3):1-16

[3] Ying Feng. Research and Application of Information Intelligent System of Modern Agricultural Based on Internet of Things [D]. Tianjin: Tianjin University, 2011

[4] Ning Wang, Naiqian Zhang, Maohao Wang. Wireless Sensors in Agriculture and Food Industry - Recent Development and Future Perspective[J]. Computer and Electronics in Agriculture, 2006,50(1):1-14

[5]Ronghui Ju, Zuorui Shen. The Greenhouse Ecological Health Call System Based on Short Message [J]. Transactions of the Chinese Society of Agricultural Engineering, 2004, 20(3):226-228.

[6]Huizhong Liu, Xiuwen Wu, Xiaoxia Feng, etc. The Application of GPRS Technology in Environmental Monitoring of Greenhouse [J]. Agricultural Equipment \& Vehicle Engineering, 2010(4):52-54 